\title{
Effect of surface defects on structure failure
}

\section{A two-scale approach}

\author{
Delphine Brancherie* — Marc Dambrine** - Grégory Vial*** \\ Pierre Villon*
}

* Laboratoire Roberval, Université de Technologie de Compiègne BP 20529, F-60205 Compiègne cedex

\{delphine.brancherie,pierre.villon\}@utc.fr

** LMAC, Université de Technologie de Compiègne BP 20529, F-60205 Compiègne cedex marc.dambrine@utc.fr

*** IRMAR, ENS Cachan Bretagne, CNRS, UEB

av. Robert Schuman F-35170, Bruz

gregory.vial@bretagne.ens-cachan.fr

ABSTRACT. This work aims to take into account the influence of boundary defects on the behaviour till rupture of structures without any fine geometrical description of the domain. This is achieved by appealing to two approaches: an asymptotic analysis of Navier equations and strong discontinuity models. We present in this work a strategy to couple the two approaches in order to provide the analysis till rupture of the structure behavior. The approach is validated on an academic example.

RÉSUMÉ. L'objectif de ce travail est de prendre en compte l'influence de la présence de défauts surfaciques sur le comportement jusqu'à rupture des structures et ce sans description fine de la géométrie des perturbations. L'approche proposée s'appuie principalement sur deux outils : une analyse asymptotique fine des équations de Navier et l'utilisation des modèles à discontinuité forte. Une stratégie de couplage des deux approches permettant l'analyse du comportement de la structure jusqu'à rupture est également présentée. L'approche est validée sur un exemple académique.

KEYWORDS: asymptotic analysis of boundary perturbations, strong discontinuity models, partition of unity method, failure.

MOTS-CLÉS : analyse asymptotique de perturbations de frontière, modèles à discontinuité forte, partition de l'unité, rupture.

DOI:10.3166/REMN.17.613-624 @ 2008 Lavoisier, Paris

REMN $-17 / 2008$. Giens 2007, pages 613 to 624 


\section{Introduction}

This paper aims to take into account the effect of surface defects on the behavior till rupture. More precisely, the objective is to evaluate the ultimate load of the structure, dealing with both small surface perturbations and localization zones development. The main feature of the proposed approach is the use of a coarse description of the geometry: neither the perturbation shape, nor a fine representation of the cohesive crack are considered.

Our approach is based on two tools. The first one - an asymptotic analysis with respect to the characteristic size of the perturbation - provides a way to evaluate how the size and shape of the defects influence the solution of the problem. The second one - a strong discontinuity approach - gives an objective description of the postpeak response until rupture. In the first two sections, we recall the main points of these techniques. Then, in a third section, we explain the way of coupling them in a numerical framework in order to compute the behavior till rupture on a mesh as coarse as possible. We present some first numerical results on a simple traction test.

\section{Effect of surface defects: asymptotic analysis}

This section presents the keypoints of the asymptotic analysis for a boundary singular perturbation in an elliptic boundary value problem. The following techniques make a strong use of linearity of the equations through a superposition principle. We consider here the Navier equations of linear elasticity. Note that the stated results do not extend easilly to inelastic material behaviors.

We describe the geometric setting we shall work within, see Figure 1 Let $\Omega_{0}$ be a bounded domain of $\mathbb{R}^{2}$, with the origin $\mathbf{0}$ being a regular point on its boundary. In the following, the first coordinate axis coincides with the tangent direction of $\partial \Omega_{0}$ at point 0.

We denote by $\omega$ another bounded domain, containing $\mathbf{0}$. The perturbed domain $\Omega_{\varepsilon}$ is obtained from $\Omega_{0}$ by removing a rescaled version of $\omega$ at size $\varepsilon$ :

$$
\Omega_{\varepsilon}=\Omega_{0} \backslash(\overline{\varepsilon \omega}) .
$$
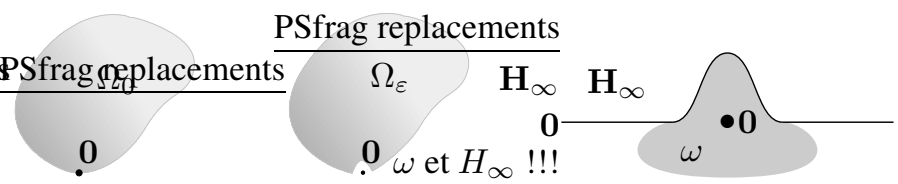

Figure 1. Initial, perturbed and blown up domains 
The problem we focus on is the following

$$
\left\{\begin{array}{l}
-\mu \Delta \mathbf{u}_{\varepsilon}-(\lambda+\mu) \operatorname{grad} \operatorname{div} \mathbf{u}_{\varepsilon}=\mathbf{f} \text { in } \Omega_{\varepsilon} \\
\mathbf{u}_{\varepsilon}=\mathbf{u}^{d} \text { on } \Gamma_{d} \\
\boldsymbol{\sigma} \cdot \mathbf{n}=\mathbf{g} \text { on } \Gamma_{t}
\end{array}\right.
$$

where $\Gamma_{d}$ and $\Gamma_{t}$ are the Dirichlet and Neumann boundaries, respectively (the perturbation point $\mathbf{0}$ naturally lies on the Neumann boundary). Besides, the volumic load $\mathbf{f}$ and the traction $\mathrm{g}$ are assumed to vanish near the perturbation. Problem [1] naturally involves two scales: the size of the structure (scale 1), and the characteristic length $\varepsilon$ of the perturbation (scale $\varepsilon$ ). At scale 1 , the domain $\Omega_{\varepsilon}$ tends to $\Omega_{0}$ as $\varepsilon \rightarrow 0$, while the limit domain at scale $\varepsilon$ (i.e. limit of $\Omega_{\varepsilon} / \varepsilon$ ) is the semi-infinite domain $\mathbf{H}_{\infty}$ defined as (see also Figure 1):

$$
\mathbf{H}_{\infty}=\left(\mathbb{R} \times \mathbb{R}^{+}\right) \backslash \bar{\omega} .
$$

We briefly recall a result from (Dambrine et al., 2007) concerning the leading terms in the asymptotic expansion of the solution $\mathbf{u}_{\varepsilon}$ to Problem [1]. This description requests two variables: $\mathbf{x}$ (slow variable) and $\mathbf{y}=\mathbf{x} / \varepsilon$ (fast variable), corresponding to scale 1 and scale $\varepsilon$, respectively. The behavior of $\mathbf{u}_{\varepsilon}$ in the fast variable relies on profiles, which are normalized functions defined in $\mathbf{H}_{\infty}$ and contribute to the expansion in variable $\mathbf{x} / \varepsilon$. Let us introduce the profile basis $\left(\mathbf{V}_{\ell}\right)_{\ell=1,2}$ as the solutions of

$$
\left\{\begin{array}{l}
-\mu \Delta \mathbf{V}_{\ell}-(\lambda+\mu) \text { grad } \operatorname{div} \mathbf{V}_{\ell}=\mathbf{0} \text { in } \mathbf{H}_{\infty} \\
\sum_{j=1}^{2} \sigma_{i j}\left(\mathbf{V}_{\ell}\right) \mathbf{n}_{j}=\mathrm{G}_{\ell, i} \text { on } \partial \mathbf{H}_{\infty}
\end{array}\right.
$$

with $\mathbf{G}_{1}=\left(\mathbf{n}_{1}, 0\right)$ and $\mathbf{G}_{2}=\left(0, \mathbf{n}_{1}\right)$ ( $\mathbf{n}_{1}$ is the first component of the outer normal on $\partial \mathbf{H}_{\infty}$ ). We emphasize that Problem [2] depends exclusively on the geometry. In particular, $\mathbf{V}_{\ell}$ is independent of the loading of Problem [1].

The expansion of $\mathbf{u}_{\varepsilon}$ takes the form

$$
\mathbf{u}_{\varepsilon}(\mathbf{x})=\mathbf{u}_{0}(\mathbf{x})-\varepsilon\left[\alpha_{1} \mathbf{V}_{1}\left(\frac{\mathbf{x}}{\varepsilon}\right)+\alpha_{2} \mathbf{V}_{2}\left(\frac{\mathbf{x}}{\varepsilon}\right)\right]+\mathbf{r}_{\varepsilon}(\mathbf{x})
$$

with

$-\mathbf{u}_{0}$ solve Problem [1] for $\varepsilon=0$ (i.e. solution in the unperturbed domain $\Omega_{0}$ ),

- the coefficients $\alpha_{1}$ and $\alpha_{2}$ are the stress values associated with $\mathbf{u}_{0}$ at point $\mathbf{0}$, namely

$$
\alpha_{1}=\sigma_{11}\left(\mathbf{u}_{0}\right)(\mathbf{0}) \quad \text { and } \quad \alpha_{2}=\sigma_{12}\left(\mathbf{u}_{0}\right)(\mathbf{0}),
$$

- the remainder $\mathbf{r}_{\varepsilon}$ satisfies the following estimate in the energy norm

$$
\left\|\mathbf{r}_{\varepsilon}\right\|_{\mathrm{H}^{1}\left(\Omega_{\varepsilon}\right)} \leq C \varepsilon^{2}
$$


As regards the computation of $\mathbf{u}_{\varepsilon}$ for small values of $\varepsilon$, Expansion [3] leads to an alternative numerical strategy to adaptative mesh refinement. Indeed the knowledge of the unperturbed solution $\mathbf{u}_{0}$ and of the profiles $\mathbf{V}_{\ell}$ gives a reasonable approximation of $\mathbf{u}_{\varepsilon}$, see further in Section 4

\section{Description of localization zones: strong discontinuity approach}

In this section, we give a short presentation of the strong discontinuity approach (SDA, see (Brancherie, 2003; Simo et al., 1993; Oliver, 1995) for more details) used to model the crack propagation. SDA aims at getting rid of mesh dependence when describing strain localization. The localization zones are taken into account through displacement discontinuity surfaces. We do not seek a fine description of such zones, but only quantify their effect on the global response of the structure. To depict the dissipative phenomenon arising at the scale of the localization zones, we introduce a traction/displacement jump law on the discontinuity surface.

The keypoint of SDA is to consider a discontinuous displacement field. If $\Gamma_{s}$ denotes the discontinuity surface, the displacement and the associated strain fields read

$$
\mathbf{u}(\mathbf{x})=\overline{\mathbf{u}}(\mathbf{x})+\overline{\overline{\mathbf{u}}} \mathcal{H}_{\Gamma_{s}}(\mathbf{x}), \quad \varepsilon(\mathbf{x})=\nabla^{\mathrm{s}} \overline{\mathbf{u}}(\mathbf{x})+(\overline{\overline{\mathbf{u}}} \otimes \mathbf{n}) \delta_{\Gamma_{s}}
$$

Two constitutive laws are built. The first one is written in the framework of classical continuum medium thermodynamics. It links the regular part $\bar{\varepsilon}=\nabla^{\mathrm{s}} \overline{\mathbf{u}}$ to the stress state in the bulk material. In the present work, we deal with a linear elastic model for this first law. The second law, written in the framework of interface thermodynamics, connects the displacement jump $\overline{\overline{\mathbf{u}}}$ to the traction $\mathbf{t}_{\Gamma_{s}}$ on the discontinuity surface $\Gamma_{s}$. It will be handled here by a surface damage model.

Numerical treatment is built on the incompatible modes method (see (Wilson et al., 1990; Ibrahimbegović et al., 1991)). A discontinuous shape function vanishing on all nodes of the element is added to the standard shape functions basis. A system of two equations is then to be solved. The first equation is the classical global weak form of the equilibrium whereas the second one corresponds to the weak form of the traction continuity on the discontinuity surface. The main feature of this last equation is to be written and solved on each localized element. We then take advantage of a static condensation step at the elements level to solve a classical linearized system with a modified stiffness matrix.

\section{Failure analysis: coupling asymptotic expansion and strong discontinuity model}

Our main objective in this work is to numerically study how the stress concentrations generated by the surface defect influence the crack propagation. Moreover, we 
want to perform such a simulation on a coarse mesh of the unperturbed domain ignoring the shape of the perturbation. To that aim, we first need to compute the solution in the perturbed domain $\Omega_{\varepsilon}$ thanks to expansion [3]. For that purpose, we use the partition of unity method (PUM, see (Melenk et al., 1996)). Then, in order to continue the computation with SDA, we transfer the resulting displacement field onto the reference mesh.

\subsection{Computation of the profiles}

In order to use PUM, we will enrich the finite element space with approximate profiles $\tilde{\mathbf{V}}_{\ell}$ corresponding to $\mathbf{V}_{\ell}$, see [2]. Their approximation is not straightforward since the domain $\mathbf{H}_{\infty}$ and its boundary are unbounded. Among all the possible available methods for such problems (boundary elements methods, infinite elements...), we have chosen, for simplicity, to use finite elements in a truncated domain. Let us fix $R>0$ relatively large and define $\mathbf{H}_{R}=\mathbf{H}_{\infty} \cap B(\mathbf{0}, R)$. We bring the vanishing condition at infinity on the artificial boundary $\partial \mathbf{H}_{R} \backslash \partial \mathbf{H}_{\infty}$. To approximate the real profile $\mathbf{V}_{\ell}$, we therefore solve

$$
\left\{\begin{array}{l}
-\mu \Delta \tilde{\mathbf{V}}_{\ell}-(\lambda+\mu) \operatorname{grad} \operatorname{div} \tilde{\mathbf{V}}_{\ell}=\mathbf{0} \text { in } \mathbf{H}_{R} \\
\sum_{j=1}^{2} \sigma_{i j}\left(\tilde{\mathbf{V}}_{\ell}\right) \mathbf{n}_{j}=\mathbf{G}_{\ell, i} \text { on } \partial \mathbf{H}_{\infty} \cap \mathbf{H}_{R} \\
\tilde{\mathbf{V}}_{\ell}=\mathbf{0} \text { on } \partial \mathbf{H}_{R} \backslash \partial \mathbf{H}_{\infty} .
\end{array}\right.
$$

The larger the radius $R$, the better the approximation. Indeed, solution of [2] decreases as $|x|^{-1}$. Another strategy allowing smaller radii, consists in using higher order absorbing boundary conditions (for a review on this subject, see (Givoli, 1991)). For the numerical implementation performed with the finite element library Mélina (see (Martin, 2007)), high order nodal elements $(\mathbb{Q} 6$, Gauss-Lobatto) are used. Since the solution $\tilde{\mathbf{V}}_{\ell}$ is expected to be localized around the origin, the mesh can be coarse elsewhere. In the results of Section 4.4 we have chosen $R=50$ and a radial mesh with $10 \times 10$ elements.

\subsection{Kinematic enrichment for the perturbed problem}

Our kinematic enrichment consists in adding to the discrete variational space the approximate profiles $\tilde{\mathbf{V}}_{\ell}$ previously computed, via partition of unity. Since the profiles $\mathbf{V}_{\ell}$ have only local influence, the enrichment is simply needed in the vicinity of the perturbation referred to as enrichment area. Namely, as suggested by [3], the approximation of the displacement field is based on

$$
\mathbf{u}_{\varepsilon}^{h}(\mathbf{x})=\mathbf{u}_{0}^{h}(\mathbf{x})-\varepsilon \sum_{\ell=1}^{2} \sum_{j \in \mathcal{J}} N^{j}(\mathbf{x})\left[\boldsymbol{\alpha}_{\ell, 1}^{j} \tilde{\mathrm{V}}_{\ell, 1}\left(\frac{\mathbf{x}}{\varepsilon}\right)+\boldsymbol{\alpha}_{\ell, 2}^{j} \tilde{\mathrm{V}}_{\ell, 2}\left(\frac{\mathbf{x}}{\varepsilon}\right)\right]
$$


with the notation

$-\mathbf{u}_{0}^{h}$ is the nodal approximation of $\mathbf{u}_{0}$ solution of [1] on the unperturbed domain,

$-\mathcal{J}$ is the set of indices of the nodes in the enrichment area,

$-N^{j}$ is the standard shape function associated with node $j$,

$-\tilde{\mathrm{V}}_{\ell, i}$ is the $i^{\text {th }}$ component of the precomputed approximate profile $\tilde{\mathbf{V}}_{\ell}$,

$-\boldsymbol{\alpha}_{\ell, i}^{j}=\left[\left(\boldsymbol{\alpha}_{\ell, i}^{j}\right)_{1},\left(\boldsymbol{\alpha}_{\ell, i}^{j}\right)_{2}\right]$ is the twodimensional vector of the degrees of freedom corresponding to the previous function $\tilde{\mathrm{V}}_{\ell, i}$.

Let us emphasize that the computation is carried out on the mesh of the unperturbed domain. The perturbation is handled through a dedicated quadrature in the enrichment area. We have added to the standard two degrees of freedom (dof) eight other dof per node in the enrichment area, leading to two types of nodes in the mesh. The treatment in a classical finite element code is twofold. The first possibility consists in adding the eight new dof to each node in the mesh imposing vanishing Dirichlet conditions outside the enrichment area. The second one could be to consider a transition zone of hybrid elements. Though more natural, the latter requires more technical adaptations.

To fit Expansion [3], we expect to find

$$
\left(\boldsymbol{\alpha}_{\ell, 1}^{j}\right)_{1}=\left(\boldsymbol{\alpha}_{\ell, 2}^{j}\right)_{2}=\alpha_{\ell} \text { and }\left(\boldsymbol{\alpha}_{\ell, 1}^{j}\right)_{2}=\left(\boldsymbol{\alpha}_{\ell, 2}^{j}\right)_{1}=0 \text { for all } j \in \mathcal{J}, \ell=1,2 .
$$

As noticed in the context of XFEM ((Chahine et al., 2007)), this problem is badly conditioned because, far from the perturbation, the added functions $\tilde{\mathbf{V}}_{\ell}$ almost belong to the standard finite element space $\operatorname{Span}\left(N^{j}\right)$. Therefore, we impose the equalities

$$
\left(\boldsymbol{\alpha}_{\ell, 1}^{j}\right)_{1}=\left(\boldsymbol{\alpha}_{\ell, 2}^{j}\right)_{2} \text { and }\left(\boldsymbol{\alpha}_{\ell, 1}^{j}\right)_{2}=\left(\boldsymbol{\alpha}_{\ell, 2}^{j}\right)_{1}
$$

as constraints by a master/slaves strategy. We then obtain the following augmented problem.

$$
\left[\begin{array}{ccc}
\mathbf{K}_{\mathbf{u u}}^{0} & \mathbf{0} & \boldsymbol{\Pi}^{\top} \\
\mathbf{K}_{\boldsymbol{\alpha u}}^{\varepsilon} & \mathbf{K}_{\boldsymbol{\alpha} \boldsymbol{\alpha}}^{\varepsilon} & \mathbf{0} \\
\mathbf{0} & \mathbf{\Pi} & \mathbf{0}
\end{array}\right]\left[\begin{array}{c}
\mathbf{u}_{0} \\
\boldsymbol{\alpha} \\
\boldsymbol{\lambda}
\end{array}\right]=\left[\begin{array}{c}
\mathbf{f}_{0} \\
\mathbf{f}_{\boldsymbol{\alpha}} \\
\mathbf{0}
\end{array}\right]
$$

where

$-\mathbf{K}_{\mathbf{u u}}^{0}$ is the usual stiffness matrix associated with the standard nodal dof of the unperturbed domain,

$-\Pi$ is the projection operator onto the constraints [8],

$-\mathbf{K}_{\alpha \alpha}^{\varepsilon}$ is the stiffness part corresponding to the added dof in the enrichment area,

$-\mathbf{K}_{\alpha \mathbf{u}}^{\varepsilon}$ is the coupling part of the stiffness matrix,

$-\lambda$ is the Lagrange multiplier associated with the constraints [8],

$-\mathbf{f}_{0}, \mathbf{f}_{\boldsymbol{\alpha}}$ are the loadings.

The exponent $\varepsilon$ in $\mathbf{K}_{\alpha \alpha}^{\varepsilon}$ and $\mathbf{K}_{\alpha \mathbf{u}}^{\varepsilon}$ indicates the use of the dedicated quadrature in the enrichment area. It consists in increasing the order of the numerical integration in 
all the elements in this area. Moreover, to integrate on $\Omega_{\varepsilon}$ instead of $\Omega_{0}$, we modify the shape of the element containing $\mathbf{0}$ to fit the perturbation, and remesh the resulting geometry to build an adapted quadrature formula.

\subsection{Field transfer}

The discrete spaces used for the PUM and SDA do not match, since they were built to describe two different phenomena (geometrical perturbation and fracture process). In order to switch from the PUM space to the SDA one (denoted $\mathcal{V}_{\text {SDA }}$ in the following), we have to project in a pertinent manner the displacement field $\mathbf{u}_{\varepsilon}^{h}$ onto the SDA approximation space. The chosen pertinency criteria are based on the strain energy. Precisely, we are led to the minimization problem:

$$
\min _{\mathbf{u} \in \mathcal{V}_{\mathrm{SDA}}} J(\mathbf{u})=\mathcal{E}\left(\mathbf{u}-\mathbf{u}_{\varepsilon}^{h}\right) \text { u.c. } \mathbf{u}_{\Gamma}=\mathbf{g}
$$

where the strain energy is defined as $\mathcal{E}(\mathbf{v})=\frac{1}{2} \int_{\Omega_{\varepsilon}} \boldsymbol{\sigma}(\mathbf{v}): \varepsilon(\mathbf{v})$.

The resulting displacement field is used as an initial value for the continuation of the computation carried out using the SDA approximation space.

\subsection{Numerical results}

The capabilities of the proposed approach are illustrated, in this section, on an academic example. We consider a rectangular specimen of dimension $200 \mathrm{~mm} \times$ $100 \mathrm{~mm}$ perforated by a semi-circular perturbation of radius $2 \mathrm{~mm}$ at point $\mathbf{0}=$ $(105 \mathrm{~mm}, 0 \mathrm{~mm})$. The specimen is submitted to a simple traction test by imposing a displacement $U$ at the free edge and convenient boundary conditions (see Figure 4.4.

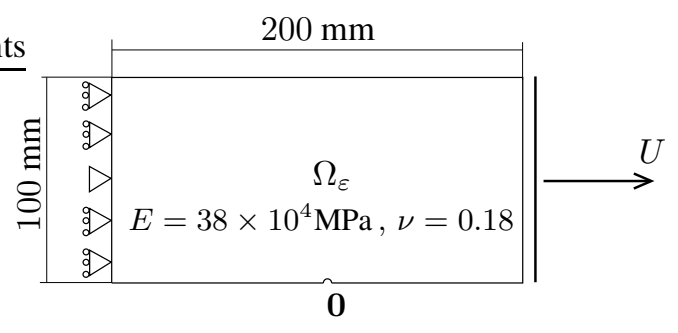

Figure 2. Dimensions and boundary conditions of the considered problem

We present further on the results obtained from the computation of the profiles, the kinematic enrichment and the field transfer leading to a full description of the rupture process of the specimen. 


\subsubsection{Multiscale correction}

To estimate the solution on the perturbed domain we need to compute an approximation $\tilde{\mathbf{V}}_{\ell}$ of the profile $\mathbf{V}_{\ell}$ (see Section 4.1). We present in Figure 3 the profile $\tilde{\mathbf{V}}_{1}$ and its derivatives on the domain of interest $\Omega_{\varepsilon}$.
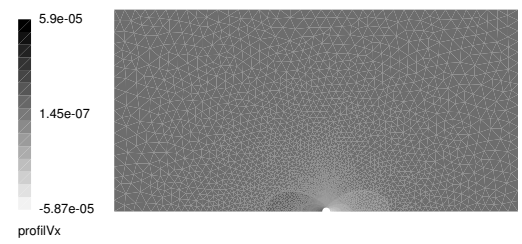

(a) $\tilde{\mathrm{V}}_{1,1}(\mathbf{y})$
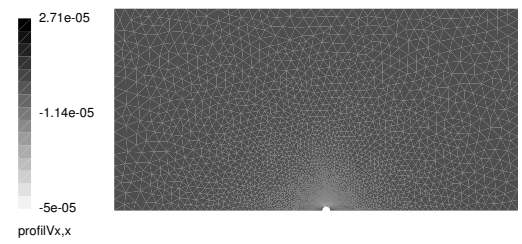

(c) $\frac{\partial \tilde{\mathrm{V}}_{1,1}(\mathbf{y})}{\partial y_{1}}$
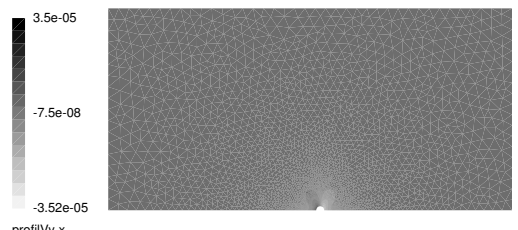

(e) $\frac{\partial \tilde{\mathrm{V}}_{1,2}(\mathbf{y})}{\partial y_{1}}$
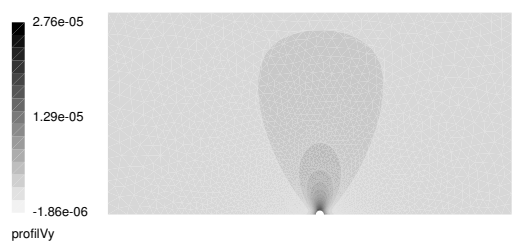

(b) $\tilde{\mathrm{V}}_{1,2}(\mathbf{y})$
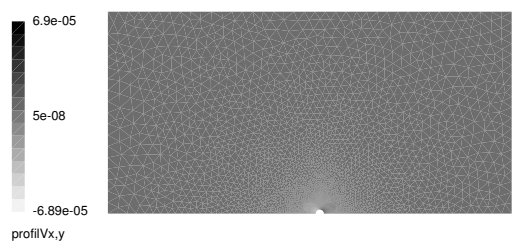

(d) $\frac{\partial \tilde{\mathbf{V}}_{1,1}(\mathbf{y})}{\partial y_{2}}$
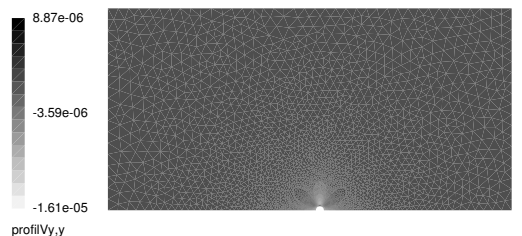

(f) $\frac{\partial \tilde{\mathrm{V}}_{1,2}(\mathbf{y})}{\partial y_{2}}$

Figure 3. Profile $\tilde{\mathrm{V}}_{1}$ and its derivatives on the domain $\Omega_{\varepsilon}$

It has to be noticed, as expected, that the influence of the perturbation is very localized around point $\mathbf{0}$. An approximation of the solution on the perturbed domain is given by Equation [3] with $\alpha_{1}=\sigma_{11}\left(\mathbf{u}_{0}\right)(\mathbf{0})$ and $\alpha_{2}=\sigma_{12}\left(\mathbf{u}_{0}\right)(\mathbf{0})=0 \mathrm{MPa}$. Figure 5 gives a map of the local relative strain energy error when comparing the result 
obtained from the superposition suggested by the asymptotic analysis and a Finite Element computation performed on a fine mesh of the real geometry (see Figure 4 . The results are given for an imposed displacement $U=1 \mathrm{~mm}$.

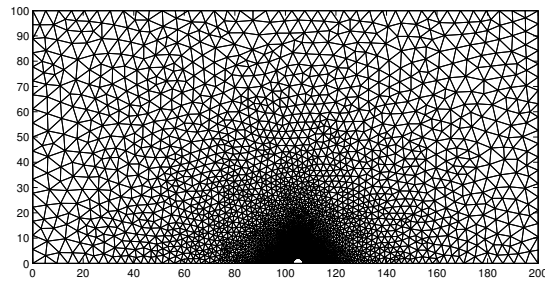

(a) Fine mesh of the real geometry

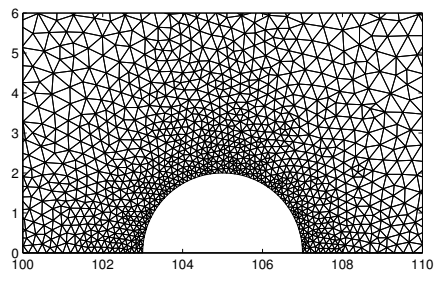

(b) Zoom around the perturbation

Figure 4. FE mesh of the real geometry

The local error on element $e$ (corresponding to area $\Omega^{e}$ ) is computed as :

$$
\operatorname{Err}(e)=\frac{\int_{\Omega^{e}} \mathcal{E}\left(\mathbf{u}_{\mathrm{AA}}-\mathbf{u}_{\mathrm{FE}}\right)}{\int_{\Omega^{e}} \mathcal{E}\left(\mathbf{u}_{\mathrm{FE}}\right)}
$$

where $\mathbf{u}_{\mathrm{AA}}$ and $\mathbf{u}_{\mathrm{FE}}$ are the displacement fields obtained from the asymptotic analysis and the finite element computation, respectively. We note that the maximum local error is about $5.4 \%$. The global strain energy error is negligible (about $5.5 \times 10^{-4 \%}$ ).

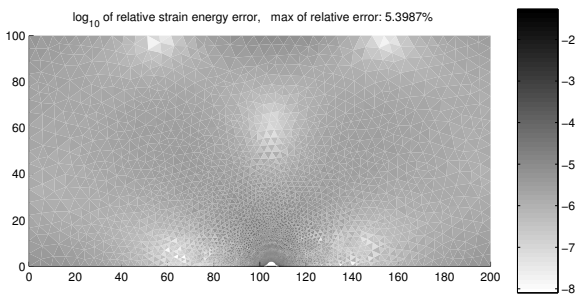

Figure 5. $\log _{10}$ of relative strain energy error

\subsubsection{Kinematic enrichment}

We give here the results obtained when performing the kinematic enrichment presented in Section 4.2 We consider a coarse mesh of the unperturbed domain (See Figure 6(a). As indicated previously the perturbation is taken into account by modifying the integration support of the element containing the perturbation. Figure 6(b) 


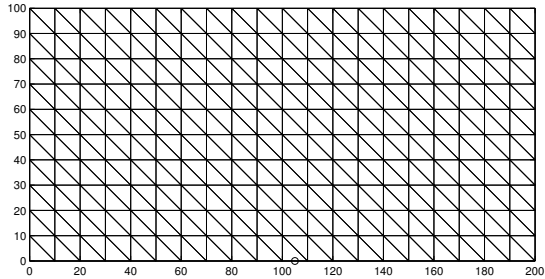

(a) Coarse mesh for enriched computation

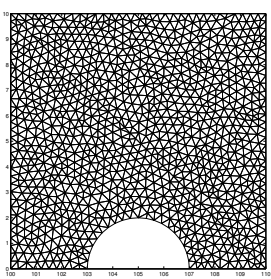

(b) Sub-domain partition for integration

Figure 6. Computation mesh and dedicated integration sub-domain

presents the quadrature mesh. The enrichment area has been chosen of radius $50 \mathrm{~mm}$ around the perturbation.

As in the previous section, we compare the results obtained by the kinematic enrichment computation and a standard Finite Element one. The map of the local strain energy error is given in Figure 7 The maximum local error is about $6.2 \%$ whereas the global energy error is here again negligible (around $8.3 \times 10^{-4 \%}$ ).

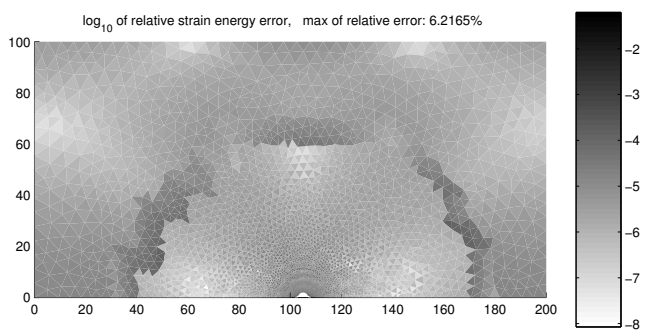

Figure 7. $\log _{10}$ of relative strain energy error

\subsubsection{Field transfer and SDA computation}

The contribution of the boundary perturbation being evaluated, the completeness of the failure analysis of the structure needs to project the obtained field to the SDA space. The obtained stress field is introduced as an initial value for the continuation of the computation. In order to verify mesh objectivity of the solution, we perform this analysis for two different meshes: a structured mesh (see Figure 6(a) and an unstructured mesh. Figure 8 presents the maximum principal stress (on which the criterion of introduction of the discontinuity is based) for the two different meshes. We note that the stress concentration induced by the perturbation is recovered even if smoothen by the transfer process. Figure 9 presents the result of the SDA computation in terms 
of crack opening and discontinuity line orientation. The discontinuities are, in both cases, initiated at the element containing the perturbation and propagate, as expected, straight to the top edge of the specimen. The mesh objectivity of the computation is illustrated on Figure 10 the responses and total dissipation evaluation obtained from the two discretisations are similar.

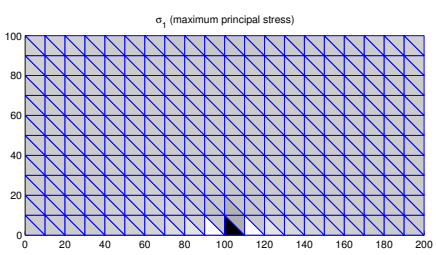

(a) Structured mesh
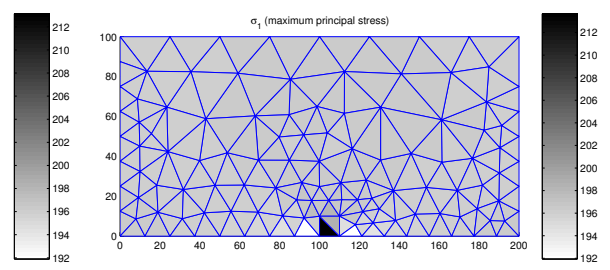

(b) Unstructured mesh

Figure 8. Maximum principal stress associated with the reconstructed displacement field $\mathbf{u}$

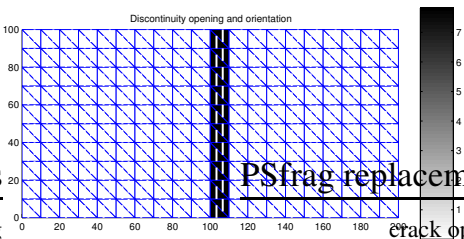

(a) Structured mesh

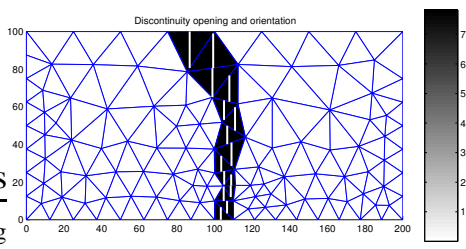

(b) Unstructured mesh

Figure 9. Discontinuity line and crack opening

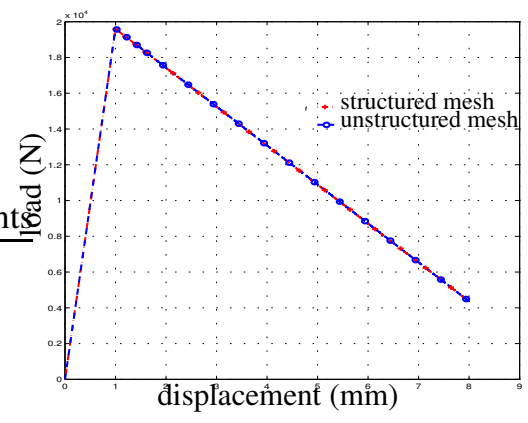

Figure 10. Load/displacement response for the two different meshes 


\section{Concluding remarks}

We have presented two methods and their coupling allowing to describe the behaviour till rupture of brittle structures. The main feature of this work is that computations are carried out at a macroscopic level while the models take into account microscopic geometrical characteristics. This is achieved thanks to an enrichment of the discrete Finite Element space with the profiles computed from the asymptotic analysis. This allows us to get rid of a fine mesh of the real geometry.

The perspectives are to consider more general loadings, geometries of the structures and multiple surface defects. We expect no major difficulties in reaching such objectives : the tools mentioned above are designed in that spirit.

\section{Acknowledgments}

This work was partially supported by the ANR (project MACADAM number JCJC06-139561). This support is gratefully acknowledged.

\section{References}

Brancherie D., Modèles "continus" et " discrets" pour les problèmes de localisation et de rupture fragile et/ou ductile, $\mathrm{PhD}$ thesis, Ecole Normale de Cachan, http://tel .archives-ouvertes.fr/tel-00132116/fr/, 2003.

Chahine E., Laborde P., Pommier J., Renard Y., Salaun M., " Study of some optimal xfem type methods", Computational Methods in Applied Sciences, 2007.

Dambrine M., Vial G., “ A multiscale correction method for local singular perturbations of the boundary”, ESAIM:M2AN, vol. 41, p. 111-128, 2007.

Givoli D., “Non-reflecting boundary conditions: a review”, J. Comp. Phys., vol. 94, n 4, p. 129, 1991.

Ibrahimbegović A., Wilson E.L., " A modified method of incompatible modes”, Communications in Applied Numerical Methods, vol. 7, p. 187-194, 1991.

Martin D., “ MÉLINA, bibliothèque de calculs éléments finis”, http://perso.univ-rennes1.fr/daniel .martin/melina, 2007.

Melenk J.M., Babuška I., " The partition of unity finite element method: Basic theory and applications", Computer Methods in Applied Mechanics and Engineering, vol. 136, p. 289314, 1996.

Oliver J., " Continuum Modelling of Strong Discontinuities in Solid Mechanics”, in D. Owen, E. Oñate, E. Hinton (eds), Computational plasticity IV. Fundamentals and Applications, Barcelone, p. 455-480, 1995.

Simo J.C., Oliver J., Armero F., “ An Analysis of Strong Discontinuity Induced by Strain Softening Solutions in Rate-Independent Solids", Journal of Computational Mechanics, vol. 12, p. 277-296, 1993.

Wilson E.L., Ibrahimbegović A., " Use of incompatible displacement modes for the calculation of element stiffnesses or stresses", Finite Elements in Analysis and Design, vol. 7, p. 229241, 1990. 